\title{
A Case Study on the Conversion of Existing Commercial Buildings into Elderly Welfare Facilities in Japan
}

\author{
H.W. Choi ${ }^{1, a}$ \\ ${ }^{1}$ Dept. of Interior Architecture and Built Environment, Yonsei University, Seoul 120-749, Korea \\ aheewonchoi0410@gmail.com
}

Keywords: Elderly Welfare Facilities, Conversion, Existing Building, Japan

Abstract. Recently, Japan with the increase in the cases of using the existing building structures for welfare purposes in recent years is displaying diversity in types of the use of the facilities prior to and following the conversion. In this Study, as the initial stage of the case survey study, cases of the conversion of the facilities such as retail stores into day service center and residential facilities for the elderly were collected and analyzed through literature review and internet search by limiting the search to Japan. In consideration of the realities in Korea in which the cases of conversion of the existing structures into welfare facilities for the elderly are forecasted to increase, it is anticipated that analysis of a diverse range of cases would provide highly useful reference at the time of conversion of use into welfare facilities for the elderly.

\section{Introduction}

According to 2011 statistics, the Korean elderly population 65 or older exceeded 5.65 million people, which accounted for $11.4 \%$ of the total population [1]. As the specific segment of the country's population is expected to increase to $15.7 \%$ of the total population in 2020, making the country an aging society, and then will reach $24.3 \%$ in 2030 , it is high time that Korea needed to prepare for a rapidly increasing aging population. Moreover, since seniors ailing from dementia or stroke are also increasing, it is urgently required to touch up the elderly care environment. By the way, as there is a limit to satisfying the sharply increasing need for elderly care facilities with newly built ones, we witness an increasing number of cases in Korea and abroad in which existing buildings are used as welfare infrastructure. As for Korea, while we see an increasing number of cases in which originally intended uses are converted to elderly welfare facilities as from schools to elderly care facilities and from small-and-medium-sized facilities to elderly care hospitals, there exist no guidelines for planning and designing elderly welfare facilities that reflect conversion of uses and change of building functions, while data that may be looked up when planning a remodeling project is not satisfactorily available. Furthermore, earlier studies are restricted to cases in which pre-conversion uses are as hospitals or closed schools, so it is the time that more various case studies were required.

Recently, Japan sees an increasing number of cases in which currently unused or low-usage existing buildings are converted to some other uses, which include those cases in which existing building resources are used to realize conversion to elderly welfare facilities. Considering the status of the country that makes us forecast an increased demand for reusing existing buildings as welfare infrastructure, the analysis of various data will serve as useful reference in expanding infrastructure for the elderly welfare facilities in Korea.

In this regard, the current study, which is meant to constitute a preliminary stage of a case study, confines its spatial scope to Japan and has performed internet search to collect for analysis cases involving conversion from some other uses to elderly welfare facilities. The purpose of this study is to contribute to setting directions for the future expansion of infrastructure of the Korean elderly welfare facilities by analyzing different cases, the outline and details of renovation, and the characteristics of current facilities.

\section{A Theoretical Consideration}

Related Research. Coming into the new millennium, we see studies published, which treat the remodeling and conversion to elderly welfare facilities. As for Korea, one may cite studies designed to assess the 
possibility of using areal facilities for local welfare, studies that examine institutions and laws that should be considered in changing uses, and studies that suggest design guidelines or apply an eco-friendly concept in remodeling into elderly facilities, while their pre-conversion uses tend to be restricted to hospitals or closed schools [2,3]. In Japan, remodeling and conversion have long been discussed, and as far as the conversion to elderly welfare facilities is concerned, pre-conversion uses include not only hospitals and schools but also dormitories, department stores, collective housing, and lodgings, thus registering greater diversity compared to Korea. Ishiguro (2004) analyzed the legal regulations for fifteen cases in which dormitories, department stores, or hospitals were converted to fee-charging nursing homes [4], while Kumazawa (2008) identified the details of construction and changes to residential performance involving cases in which collective housing, dormitories, or lodgings were converted to rental homes [5]. City Bureau of the Ministry of Land, Infrastructure, Transport and Tourism of Japan (2012) disclosed case studies on unoccupied downtown buildings and their renewal, and the cases covered by the report included conversion into the elderly welfare facilities [6].

\section{Results}

Overview of the cases. Those treated are three cases in which existing buildings were converted in small-and-medium-sized Japanese cities; as for their pre-conversion uses, Cases A and B were department stores, and Case $\mathrm{C}$ was a commercial building that included stores, restaurants, karaoke, and bowling alley lane. For Cases A and B, the entire building was converted while for Case $\mathrm{C}$, the ground-floor stores and $4 \mathrm{~F}$ $\& 5 \mathrm{~F}$ bowling lane was regenerated into elderly welfare facilities $[6,7,8,9]$.

Characteristics of Cases in Which Buildings Are Remodeled. This section describes key changes involved in remodeling, details, problems, and key issues.

Case A. Previously a major department store in the downtown district of Sano City, it was evacuated in 1996 with pullout due to bad business. Afterwards, the building remained empty and tenantless for several years, and a doctor who was looking in downtown area for a site for a project for elderly people who needed care bought it and opened in 1999 rental homes and facilities compatible with care services. In selecting a building where to open a facility for elderly people, the site conditions with various hospitals and stores close by were considered, moreover, the remodeling work focused on improving the light availability that was poor because it was previously used as a department store. Also, by increasing the breadth of the hallways to overcome the limitations of the double-loaded corridor, they were able to promote residents' health and use the space for communication.

Case B. With the pullout of a department store, which served as a symbol of the region due to its location right across from the station, the building owner sought new uses for it and remodeled it into a welfare facility. The remodeling was done mostly on $2 \mathrm{~F}$ through $6 \mathrm{~F}$ to provide paid nursing home with care, short stay, and day care. In order to overcome the shortcomings of the small number and size of the openings due to its previous use as a commercial building and thereby improve residential performance for elderly people, openings were added to the sides of the building, while skylight was installed on rooftop to secure natural lighting for residential space. Also, reinforcement work was done to strengthen earthquake-proof and structural performance.

Case C. In this case, a medical foundation, which had been looking to open a paid nursing home in downtown area, came to rent a building that was left by a moving bowling alley lane and regenerate it into a welfare facility. As there was community opposition in the planning stage to the opening of a welfare facility instead of a customer-accommodating or commercial facility in the existing building, they sought harmony with the local community with a remodeling work that installed on ground a floor welfare products shop, welfare counseling center etc. and adjusting the positions of openings. In 2009, apart from the nursing home, a day-care for children with impairment was installed on the remodeled ground floor and starting in 2011, the 
whole ground floor has been used for a day care for elderly people. Cargo elevators, which were installed in existing buildings, are of appropriate size to accommodate mobile beds and are refurbished for continued use.

Table 1 General Conditions of Cases

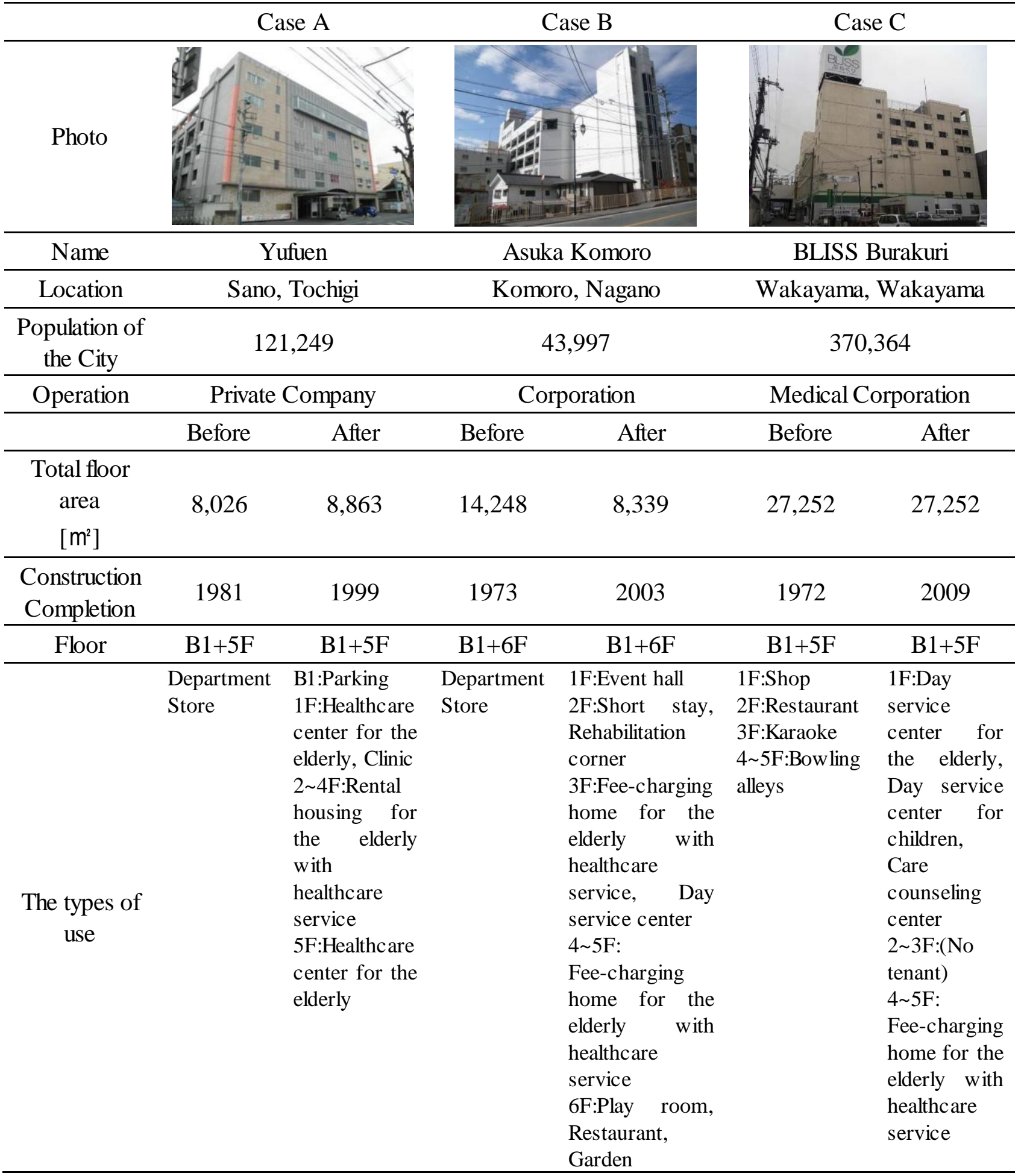

\section{Discussion and Conclusion}

This study selects three Japanese cases in which existing building previously used for commercial purposes are converted to elderly welfare facilities to analyze the changes of their physical environment and key issues involved in the remodeling work. The study has taken cases in which commercial buildings that were previously used as a department store, a store, or a bowling alley lane were converted to a paid nursing home 
or a day care, and examined problems involved in their remodeling into elderly welfare facilities and design factors for their improvement. Through the cases treated by this study, I have seen that one of the important projects is to improve the light availability that was previously insufficient, as the building was used as a large commercialoperator like a department store. I have also found wisdom for running a program and organizing space to make sure that the facility does not find itself unwanted and rather achieve harmony with the neighborhood.

Further work will have to be done through on-site investigations designed to get a real idea about the actual usage. And it is believed that we need to standardize the changes to spatial composition through collecting a sufficient number of cases, closely study problems and precautions in converting buildings to elderly welfare facilities, and conduct post-occupancy evaluation on users of converted elderly welfare facilities. Also, I am going to continue studies that review trials and errors related to such cases. And I hope that the accumulated research results will broaden the scope of remodel ability and will be used as reference that can contribute to setting directions for planning elderly welfare facilities that tap into existing buildings and expanding regional welfare.

\section{Acknowledgements}

This research was supported by Basic Science Research Program through the National Research Foundation of Korea(NRF) funded by the Ministry of Education(2014R1A1A2056002).

\section{References}

[1] Statistics Korea: Future Population Projection (2010).

[2] M.R. Chung: A Fundamental Study on the Scheme of Converting Small Hospitals and Doctors' Offices into Nursing Homes in Metropolitan Areas, Journal of the architectural institute of Korea planning \& design, Volume 26, Number 6 (2010), p.3-10

[3] J.Y. Kim and J.K. Lee: A Study on the Planning Type of Elderly Welfare Facilities by Remodeling Closed School, Journal of the Korean Housing Association, Volume 24, Number 6 (2013), p.69-76

[4] K. Ishiguro and S. Nakayama: A Survey on Building Conversion to the Living Facilities for Elderly People : A Feasibility Study on Building Conversion, Summaries of technical papers of Annual Meeting Architectural Institute of Japan, E-2 (2004), p.479-480

[5] Y. Kumazawa, K. Ikuta, S. Murakami and T. Yamashita: A Study on the Conversion from Another Type of Building into the Rental Housing Exclusively for the Elderly, Journal of architecture and planning, Volume 73, Number 633 (2008), p.2317-2324.

[6] City Bureau of the Ministry of Land, Infrastructure, Transport and Tourism of Japan: Report of Case Studies on Unoccupied Downtown Buildings and Their Renewal (2012)

[7] Information on http//www.yufuen.co.jp

[8] Information on http:/www.tbcss.co.jp/wp/?page_id=6

[9] Information on http://www.bliss-burakuri.com 